\title{
A Prospective, Comparative, Clinical Study to Evaluate the Safety and Efficacy of Two Different 0.1\% Riboflavin Solutions Used in Collagen Crosslinking Treatment for Patients with Keratoconus
}

\author{
Sheetal Brar (D) \\ Sri Ganesh (D) \\ Shilpa S Reddy \\ Nagesh BN \\ Dhwni Shahanand
}

Department of Phaco-Refractive Surgery, Nethradhama Superspecialty Eye Hospital, Bangalore, Karnataka, India
Correspondence: Sheetal Brar Nethradhama Superspeciality Eye Hospital, 256/I4, Kanakapura Main Road, 7th Block, Jayanagar, Bengaluru,

Karnataka, 560070, India

Tel +919591002092

Email brar_sheetal@yahoo.co.in
Purpose: To compare the safety and efficacy of $0.1 \%$ riboflavin in two different solutions which is used in corneal collagen crosslinking (CXL) for the treatment of keratoconus.

Methods: This was a prospective, randomized, comparison study which included 100 eyes of 61 patients with progressive keratoconus who underwent CXL with riboflavin $0.1 \%$ solution as a photosensitizer, using the standard Dresden protocol of using $3 \mathrm{~mW} / \mathrm{cm}^{2} \mathrm{UV}$ A irradiation for 30 minutes which corresponds to a total energy of $5.4 \mathrm{~J} / \mathrm{cm}^{2}$. The recruited patients were divided into 2 groups ie, Flavin Group and Peschke-D group by computer generated randomization. Postoperative examinations were conducted on 1 day, 1 month, 3 months, 6 months and 12 months after the crosslinking.

Results: For both groups, the mean manifest spherical equivalent (SE), astigmatism, best corrected distance visual acuity, keratometry values, thinnest pachymetry values, demarcation line depth and endothelial cell density preoperatively and at postoperative 12 months were comparable with no statistically significant differences. At 12 months postoperatively, $62 \%$ of eyes in the Flavin group, and $68 \%$ of eyes in the Peschke-D group had postoperative manifest SE of within \pm 1.00 D. During UV-A exposure, the cornea in the Flavin group showed intraoperative thinning of 112 microns (27\%) as compared with a thinning of 108.12 microns $(26.5 \%)$ observed in the Peschke group $(p=1.67)$ from the initial pachymetry readings. No eye in either group had any immediate or long-term postop vision threatening complications such as infectious keratitis, corneal melt, non-resolving corneal oedema or endothelial decompensation.

Conclusion: Both riboflavin solutions were equally safe and effective in the management of progressive keratoconus, and resulted in similar changes in terms of mean manifest spherical equivalent (SE), astigmatism, best corrected distance visual acuity, keratometry values, thinnest pachymetry values, demarcation line depth and endothelial cell density at the end of 12 months postoperatively.

Trial Registration Number: Ctri/2019/11/021841 (Www.ctri.nic.in).

Keywords: keratoconus, collagen cross linking, CXL, riboflavin, Flavin, Peschke

\section{Introduction}

There has been a major breakthrough in management of keratoconus by preventing the progression of keratoconus with the procedure of corneal collagen crosslinking with Riboflavin $0.1 \%$ and Ultraviolet A (UVA) light. ${ }^{1,2}$ This treatment when offered to patients with progressive Keratoconus $(\mathrm{KCN})$ and treated at the right stage of the 
disease, can help in prevention of further progression of keratoconus, especially in younger patients, reducing the morbidity, and improving their vision by post procedure visual rehabilitation. Until recently most Keratoconus treatments focused on correcting the refractive errors induced by the corneal deformation or changing the corneal geometry as in corneal transplantation, but the only treatment that aims to stop or decrease the rate of progression of keratoconus is Corneal Collagen crosslinking (CXL). ${ }^{3}$ It is a procedure that uses Ultraviolet A (UVA) light in conjunction with Riboflavin $0.1 \%$ as a photosensitizer to add additional covalent bonds between collagen fibers and thereby increasing corneal biomechanical resistance. ${ }^{4,5}$ It is also nowadays used for stabilization of corneal tissue, which is accompanied by a reduction in the simulated keratometry (simK) values and a modest improvement in visual acuity. A study conducted by Derakhshan et al showed that maximum and mean sim $\mathrm{K}$ values decreased by $0.65 \mathrm{D}$ and 0.51 D respectively $(\mathrm{P}<0.05)$ in $77 \%$ of the treated eyes. These results were observed at three months and remained stable up to six months. ${ }^{6}$ Another study by Agarwal et al also showed that maximum $\mathrm{K}$ value decreased by a mean of $2.47 \mathrm{D}$ in $54 \%$ of patients and remained stable in $38 \%$ of treated eyes. $^{7}$

Different formulations of riboflavin are available in the market and have been evaluated in previous studies. ${ }^{8}$ Recently, a new formulation - Flavin Isotonic Riboflavin Ophthalmic Solution 0.1\% - Flavin (Biotech Ophthalmics Pvt. Ltd., Ahmedabad, Gujarat, India) was introduced in the market to be used for the purpose of collagen cross linking. To our knowledge, this solution has not been previously evaluated. The current study was thus undertaken with the purpose of evaluating and comparing the safety and efficacy of new Flavin isotonic riboflavin ophthalmic solution along with Peschke-D Standard Riboflavin Solution, which has been evaluated earlier in clinical trials and in market for a long time. ${ }^{9}$

\section{Methodology}

This prospective, randomized, comparative clinical trial was undertaken to evaluate the safety and effectiveness of Flavin Isotonic Riboflavin Ophthalmic Solution 0.1\% (Biotech Ophthalmics Pvt. Ltd.) versus that of Peschke ${ }^{\circledR}$ D Standard Riboflavin Solution with Dextran (PESCHKE Trade GmbH, Switzerland) for performing Corneal Crosslinking (CXL) treatment for patients with Keratoconus. The study was approved by the institutional ethics committee of Nethradhama Superspeciality Eye
Hospital, Bangalore and adhered to the tenets of the Helsinki Declaration. Written informed consent was obtained from all patients participating in the study.

\section{Inclusion Criteria}

Patients presenting with a unilateral/bilateral case of keratoconus with documented progression (as per previously defined criteria), patients $\geq 18$ years of age, mean keratometric readings $\leq 55 \mathrm{D}$ on pentacam, patients with clear central cornea without apical scarring, corneal thickness $\geq 400$ microns at the thinnest point, and patients willing to sign informed consent and come for all postoperative follow-up procedures as defined in study protocol were included in the study.

\section{Exclusion Criteria}

Eyes classified as either normal, atypical normal (except corneal ectasia) or keratoconus suspect on the severity grading scheme, a history of previous corneal surgery or the insertion of intrastromal ring implantation in the eye(s) to be treated, previous corneal or intraocular surgery, pellucid marginal corneal degeneration, eyes which are aphakic or pseudophakic and do not have a UV blocking lens implanted, a history of delayed epithelial healing, patients with nystagmus or any other condition that would have prevented a steady gaze during the CXL treatment or other diagnostic tests, and pregnant and lactating women were excluded from the study.

\section{Composition of Riboflavin Solutions Evaluated in the Study}

\section{Flavin}

Sterile, preservative-free ophthalmic solution containing Riboflavin $0.1 \%$ and $20 \%$ Dextran P500 in saline buffer.

\section{Peschke-D}

Sterile, preservative-free ophthalmic solution containing Riboflavin (Vitamin B2) $0.1 \%$ and 20\% dextran 500.

\section{Surgical Procedure}

The whole procedure was conducted under sterile conditions in the operating room. Proparacaine $0.5 \%$ eye drops were instilled twice at an interval of 5 minutes for perioperative local anaesthesia. The central $8-10 \mathrm{~mm}$ of the corneal epithelial debridement was done using a blunt knife. As a photosensitizer, riboflavin $0.1 \%$ solution (either Flavin or Peschke-D) consisting of Riboflavin Sodium Phosphate $0.1 \%$ in dextran- P-500 20\% solution 
was applied every 2 minutes for a minimum soaking period of 30 minutes, after which the penetration of the dye into the anterior chamber was confirmed using a portable slit lamp. Following this, the eye was irradiated with UV-A radiation using CL-UVR Rapid (Appasamy Associates, Chennai, India). Before each treatment, the desired irradiance of $3 \mathrm{~mW} / \mathrm{cm}^{2}$ was controlled with a UVA meter at 5-cm distance and regulated with the potentiometer. The patient's cornea was then irradiated with the UV-A-light diodes $(370 \mathrm{~nm})$ for 30 minutes using $3 \mathrm{~mW} / \mathrm{cm}^{2}$ irradiance, which corresponds to a dose of $5.4 \mathrm{~J} / \mathrm{cm}^{2}$. During the UV irradiation, $0.1 \%$ riboflavin drops were instilled every 2 minutes and centration of the treatment monitored continuously by the surgeon and adjusted when needed.

During the procedure, intraoperative pachymetry was measured using a handheld ultrasound system (Tomey ${ }^{\circledR}$ SP-100, Tomey, Nagayo, Japan) preoperatively, after removal of epithelium, after 30 minutes of riboflavin saturation and after the completion of UV-A radiation exposure for both the groups. After the treatment, a bandage contact lens was applied for 4 days or until reepithelization had completed.

Postoperative medication regimen was similar in both groups and consisted of topical $0.3 \%$ Ofloxacin (Exocin ${ }^{\circledR}$, Allergan, Irvine, USA) 4 times/day for 10 days, $0.1 \%$ prednisolone acetate eye drops (Pred Forte ${ }^{\circledR}$, Allergan) 4 times/day for 4 weeks (tapering weekly), and lubricants 4 times/day for 4 weeks or more.

Postoperative examinations were conducted on postoperative day 1, 2 weeks, 1 month, 3 months, 6 months and 12 months. From 2 weeks onwards, slit lamp examination and assessment of UDVA, CDVA, manifest refraction, corneal topography by Pentacam HR and evaluation of haze grading were performed. In addition to the above, Anterior Segment OCT (Optovue, Fremont, USA) was also performed to study the interface quality and depth of demarcation.

\section{Statistical Analysis}

SPSS software for Windows version 17.0.0 (IBM Corp., Armonk, NY) was used for statistical analysis. Independent sample $t$-test was used for intergroup comparison and paired $t$-test was used for intragroup comparison of means. A p-value of 0.05 or less was considered statistically significant.

\section{Results}

One hundred eyes from 61 patients with unilateral or bilateral keratoconus were randomized based on a computer generated method to receive collagen cross linking treatment with either of the two isotonic riboflavin $0.1 \%$ solutions, ie, Flavin or Peschke-D, with 50 eyes in each group.

For both groups, the values of mean preoperative manifest SE, sphere, cylinder, CDVA, steep and flat keratometry readings and thinnest pachymetry were comparable with no statistically significant differences; ( $p$-values $>0.05$ for all parameters) as shown in Table 1 . Table 2 shows the postoperative outcomes for both groups at postop 12 month follow-ups. Both groups were comparable in terms of postop UDVA, CDVA, SE, cylinder, flat and steep keratometry readings, maximum keratometry, thinnest pachymetry and mean depth of demarcation line (Figures 1 and 2).

Tables 3 and 4, individually show the results of Flavin and Peschke-D groups, respectively. As shown in Tables 3 and 4, depicting individual results preoperative and 12month postoperative findings of Flavin and Peschke-D groups respectively, in both the groups, the preoperative values compared to the 12 month postoperative values and no significant change was observed for manifest SE, astigmatism, keratometry readings, thinnest pachymetry and endothelial cell density at 12 months postop.

\section{Intraoperative Pachymetry Changes}

Before removal of the corneal epithelium, the mean ultrasound pachymetry measurements were 460 and 452 microns in the Flavin group and Peschke group,

Table I Demographics and Baseline Preoperative Parameters of Both Groups

\begin{tabular}{|l|l|l|l|}
\hline Parameters & $\begin{array}{l}\text { Flavin }(\mathbf{n}=\mathbf{5 0}) \\
\text { Mean } \pm \text { SD }\end{array}$ & $\begin{array}{l}\text { Peschke D }(\mathbf{n}=\mathbf{5 0}) \\
\text { Mean } \pm \text { SD }\end{array}$ & p-value \\
\hline Age (years) & $25 \pm 4.49$ & $24.12 \pm 5.23$ & 0.33 \\
Sph (D) & $-1.29 \pm 1.5$ & $-1.22 \pm 82$ & 0.80 \\
Cyl (D) & $-2.285 \pm 1.62$ & $-2.20 \pm 1.59$ & 0.44 \\
SE (D) & $-2.43 \pm 1.66$ & $-2.32 \pm 1.92$ & 0.92 \\
UDVA (logMAR) & $0.54 \pm 0.36$ & $0.55 \pm 0.37$ & 0.90 \\
CDVA (logMAR) & $0.23 \pm 0.25$ & $0.21 \pm 0.20$ & 0.61 \\
ECD (cells/mm $\left.{ }^{2}\right)$ & $2695.92 \pm 243.38$ & $2730.08 \pm 275.54$ & 0.45 \\
Thinnest pachymetry & $466.78 \pm 34.87$ & $457.5 \pm 35.52$ & 0.11 \\
(microns) & & & \\
KI (D) & $45.49 \pm 2.31$ & $45.38 \pm 2.92$ & 0.82 \\
K2 (D) & $49.11 \pm 3.51$ & $48.60 \pm 4.02$ & 0.59 \\
KMEAN (D) & $47.20 \pm 2.60$ & $48.63 \pm 4.0$ & 0.68 \\
\hline
\end{tabular}

Abbreviations: Sph, sphere; Cyl, cylinder; SE, spherical equivalent; D, Diopter; UDVA, Uncorrected distance visual acuity; CDVA, Corrected distance visual acuity; ECD, Endothelial cell count; K, Keratometry. 
Table 2 Visual and Refractive Outcomes of Both Groups at 12 Months

\begin{tabular}{|l|l|l|l|}
\hline Parameters & $\begin{array}{l}\text { Flavin } \\
(\mathbf{n}=\mathbf{5 0})\end{array}$ & $\begin{array}{l}\text { Peschke } \mathbf{D} \\
\mathbf{( n = 5 0 )}\end{array}$ & p-value \\
\hline Sph (D) & $-1.28 \pm 1.95$ & $-1.1 \pm 1.70$ & 0.55 \\
Cyl (D) & $-2.29 \pm 1.41$ & $-2.12 \pm 1.40$ & 0.53 \\
SE (D) & $-2.4 \pm 1.95$ & $-2.16 \pm 1.70$ & 0.44 \\
UDVA (logMAR) & $0.65 \pm 0.38$ & $0.57 \pm 0.36$ & 0.24 \\
CDVA (logMAR) & $0.22 \pm 0.22$ & $0.16 \pm 0.15$ & 0.08 \\
ECD (cells/mm ${ }^{2}$ ) & $2674.38 \pm 236$ & $2696.3 \pm 225.2$ & 0.58 \\
Thinnest pachymetry (microns) & $460.1 \pm 32.35$ & $453.76 \pm 36.06$ & 0.29 \\
KI (D) & $45.44 \pm 2.93$ & $45.26 \pm 2.89$ & 0.76 \\
K2 (D) & $48.976 \pm 3.84$ & $48.22 \pm 3.84$ & 0.31 \\
KMEAN (D) & $46.99 \pm 3.11$ & $46.72 \pm 3.30$ & 0.55 \\
\hline
\end{tabular}

Abbreviations: Sph, sphere; Cyl, cylinder; SE, spherical equivalent; D, Diopter; UDVA, Uncorrected distance visual acuity; CDVA, Corrected distance visual acuity; ECD, Endothelial cell count; K, Keratometry.

respectively $(p=0.08)$. Immediately after removal of the epithelium, the mean pachymetry was 414 and 408 microns, respectively ( $\mathrm{p}=0.17$ ). After 30 -minute administration of riboflavin solution (Flavin and Peschke D), the mean pachymetry was 302 and 299.88 microns in the Flavin and Peschke D groups, respectively $(\mathrm{p}=0.37)$. Postoperatively, immediately after UVA light administration, the mean pachymetry was 298 and 296 microns, respectively. Thus, during riboflavin soaking time, the Flavin group cornea thinned by 112 microns $(27 \%)$ on average compared with thinning of 108.12 microns $(26.5 \%)$ in the Peschke group ( $\mathrm{p}=1.67)$.

The mean changes at the thinnest pachymetry at the end of 6 months and 12 months postoperatively were $-6 \mu$ (p-0.006) and $-6.68 \mu(p=0.002)$, respectively in the Flavin group. For the Peschke-D group, mean reduction of thinnest pachymetry values were -2.46 and $-3.74 \mu$ at 6 months $(p=0.06)$ and 12 months $(\mathrm{p}=0.01)$ postoperatively, respectively (Table 5 ).

\section{Efficacy}

A cumulative Snellen Visual Acuity of 20/40 or better was achieved in $30 \%$ of eyes in the Flavin group, versus $38 \%$ of eyes in the Peschke-D group (Figure 3).

The mean UDVA at 12 months was $0.65 \pm 0.38$ and 0.56 \pm 0.35 LogMAR in the Flavin and Peschke-D groups respectively, which was comparable between the two groups $(\mathrm{p}=0.24)$, Table 2 .

\section{Safety}

All eyes in both the groups achieved CDVA of 20/200 or better postoperatively (Figure 3). 92\% of eyes in the Flavin group versus $88 \%$ of eyes in the Peschke-D group had no change in the CDVA at 12 months postoperatively. $4 \%$ and $8 \%$ of eyes in Flavin and Peschke-D groups respectively had a gain of 1 line of CDVA. However, a minor percentage of eyes $(4 \%$ in Flavin and $4 \%$ in

12 Months Post-op

Pre-op

Difference

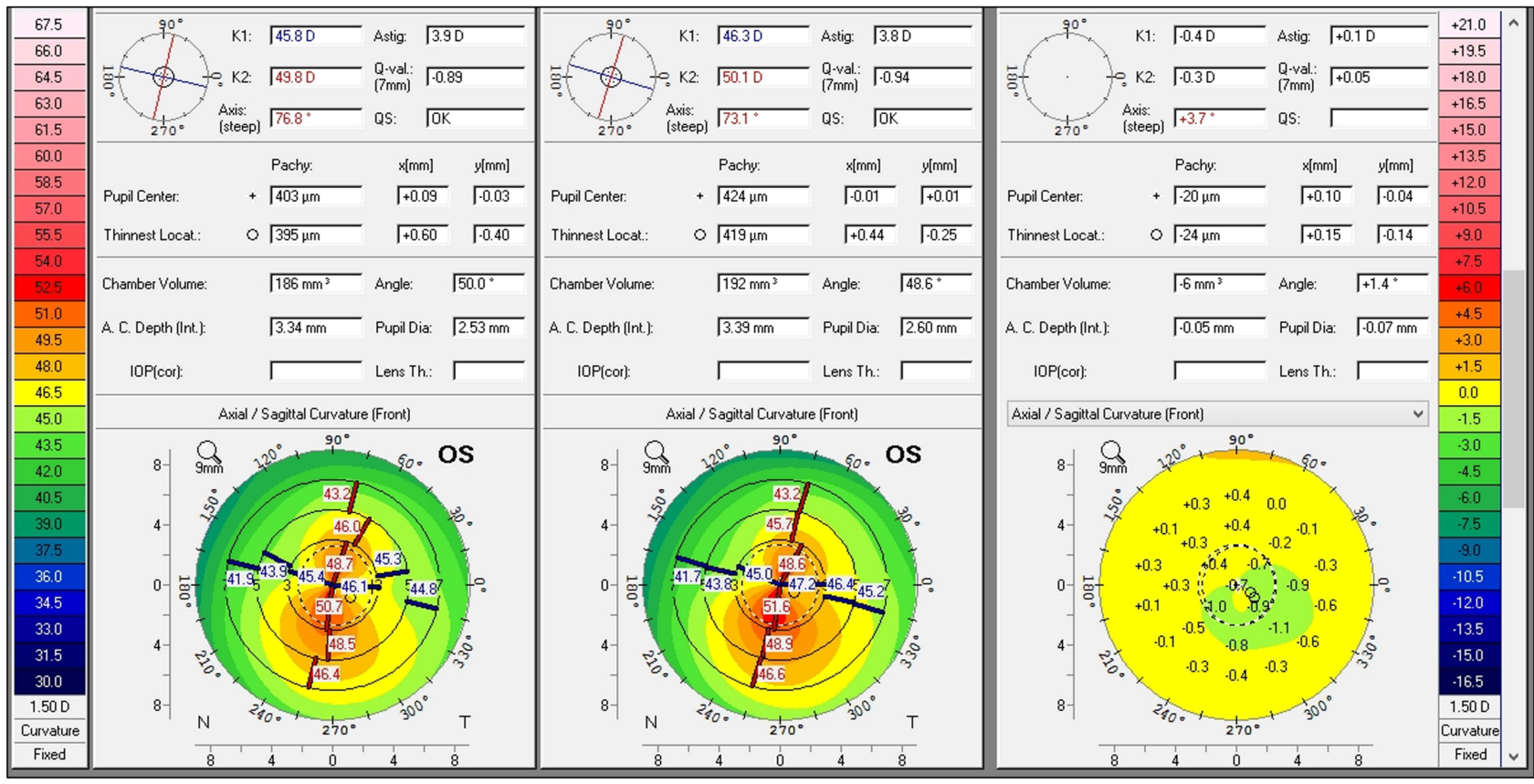

Figure I Difference map (12 months preop) showing 12 months outcome of a patient who underwent cross linking treatment using Flavin riboflavin dye. 
12 Months Post-op

Pre-op

Difference

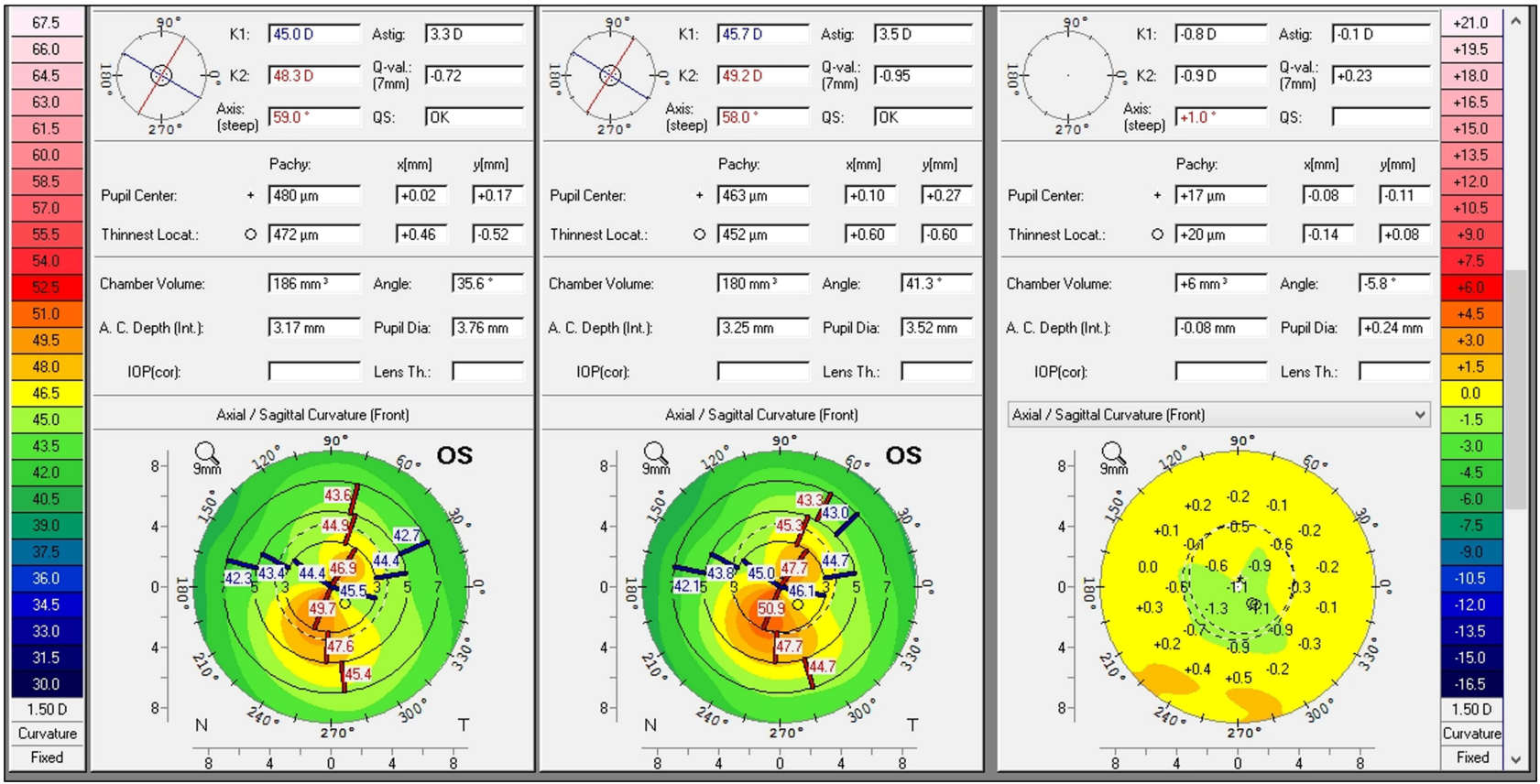

Figure 2 Difference map (12 months preop) showing 12 months outcome of a patient who underwent cross linking treatment using Peschke-D riboflavin dye.

Peschke-D groups) had loss of 1 line of CDVA at 12 months (Figure 4).

\section{Refraction, Predictability and Stability}

At 12 months, $62 \%$ of eyes in the Flavin group, and $68 \%$ of eyes in the Peschke-D group had postop refractive SE of within $\pm 1.00 \mathrm{D}$ (Figure 5). In terms of the refractive astigmatism, $26 \%$ and $32 \%$ of eyes in the Flavin and Peschke-D groups were within $\pm 1.00 \mathrm{D}$ (Figure 6).
There was no significant change in the mean postop SE in both groups, which showed good stability at 12 months compared to preop. The mean SE slightly reduced from $-2.43 \mathrm{D}$ to $-2.4 \mathrm{D}$ in the Flavin group and $-2.32 \mathrm{D}$ to $-2.16 \mathrm{D}$ in the Peschke-D group, however the difference was not statistically significant (Figure 7).

\section{Depth of Demarcation Line}

The mean depth of demarcation line at 1-month postop in the Flavin group was $256.27 \pm 61.49 \mu$ and in the Peschke-D

Table 3 Visual and Refractive Outcomes of Flavin Group Over 12 Months

\begin{tabular}{|c|c|c|c|c|c|c|}
\hline Parameters & Preop & I Month & 3 Months & 6 Months & 12 Months & p-value \\
\hline Sph (D) & $-1.29 \pm 1.5$ & $-1.275 \pm 1.91$ & $-1.25 \pm 1.98$ & $-1.29 \pm 2$ & $-1.28 \pm 1.98$ & 0.99 \\
\hline Cyl (D) & $-2.85 \pm 1.69$ & $-2.36 \pm 1.44$ & $-2.25 \pm 1.48$ & $-2.29 \pm 1.43$ & $-2.29 \pm 1.43$ & 0.99 \\
\hline SE (D) & $-2.43 \pm 1.68$ & $-2.45 \pm 2.06$ & $-2.4 \pm 2.07$ & $-2.4 I \pm 2.0 I$ & $-2.4 \pm 1.96$ & 0.99 \\
\hline UDVA (logMAR) & $0.54 \pm 0.35$ & $0.67 \pm 0.40$ & $0.64 \pm 0.37$ & $0.65 \pm 0.38$ & $0.65 \pm 0.38$ & 0.99 \\
\hline CDVA (logMAR) & $0.23 \pm 0.25$ & $0.24 \pm 0.24$ & $0.24 \pm 0.25$ & $0.23 \pm 0.23$ & $0.22 \pm 0.22$ & 0.99 \\
\hline ECD (cells/mm²) & $2695.92 \pm 243.37$ & $2567.32 \pm 205.39$ & $2642.5 \pm 292.43$ & $2660.24 \pm 239.71$ & $2674.38 \pm 236$ & 0.09 \\
\hline Thinnest pachymetry (microns) & $466.78 \pm 34.87$ & $463.2 \pm 35.18$ & $462.82 \pm 34.03$ & $460.78 \pm 32.61$ & $460.1 \pm 32.35$ & 0.87 \\
\hline $\mathrm{KI}(\mathrm{D})$ & $45.49 \pm 2.31$ & $45.39 \pm 2.39$ & $45.44 \pm 2.96$ & $45.40 \pm 2.95$ & $45.44 \pm 2.93$ & 0.99 \\
\hline K2 (D) & $49.11 \pm 3.51$ & $48.99 \pm 3.53$ & $47.13 \pm 3.85$ & $48.98 \pm 3.70$ & $48.97 \pm 3.84$ & 0.99 \\
\hline KMean(D) & $47.20 \pm 2.60$ & $47.16 \pm 2.79$ & $47.20 \pm 3.21$ & $47.04 \pm 3.1$ & $47 \pm 3.11$ & 0.99 \\
\hline DLD (microns) & 0 & $256.27 \pm 61.49$ & $255.45 \pm 61.09$ & 0 & 0 & - \\
\hline
\end{tabular}

Abbreviations: Sph, sphere; Cyl, cylinder; SE, spherical equivalent; D, Diopter; UDVA, Uncorrected distance visual acuity; CDVA, Corrected distance visual acuity; ECD, Endothelial cell count; K, Keratometry; DLD, Demarcation line depth. 
Table 4 Visual and Refractive Outcomes of Peschke Group Over 12 Months

\begin{tabular}{|l|l|l|l|l|l|l|}
\hline Parameters & Preop & I Month & 3 Months & $\mathbf{6}$ Months & I2 Months & p-value \\
\hline Sph (D) & $-1.22 \pm 8 I$ & $-1.15 \pm 1.68$ & $-1.20 \pm 1.72$ & $-1.18 \pm 1.76$ & $-1.1 \pm 1.69$ \\
Cyl (D) & $-2.20 \pm 1.59$ & $-2.14 \pm 1.28$ & $-2.08 \pm 1.41$ & $-2.15 \pm 1.54$ & $-2.13 \pm 1.55$ & 0.99 \\
SE (D) & $-2.32 \pm 1.93$ & $-2.21 \pm 1.62$ & $-2.24 \pm 1.76$ & $-2.25 \pm 1.79$ & $-2.16 \pm 1.72$ & 0.99 \\
UDVA (logMAR) & $0.55 \pm 0.37$ & $0.59 \pm 0.40$ & $0.59 \pm 0.39$ & $0.56 \pm 0.35$ & $0.5 \pm 0.35$ & 0.96 \\
CDVA (logMAR) & $0.21 \pm 0.2$ & $0.21 \pm 0.17$ & $0.2 \pm 0.18$ & $0.18 \pm 0.17$ & $0.16 \pm 0.14$ & 0.51 \\
ECD (cells/mm ${ }^{2}$ ) & $2730.08 \pm 275.54$ & $2698.8 \pm 451.86$ & $2700.62 \pm 241.01$ & $2689.26 \pm 235.67$ & $2696.3 \pm 225.2$ & 0.93 \\
Thinnest pachymetry (microns) & $457.5 \pm 35.52$ & $453.9 \pm 36.27$ & $453.42 \pm 36.09$ & $455.04 \pm 35.16$ & $453.76 \pm 36.06$ & 0.97 \\
KI (D) & $45.38 \pm 2.92$ & $45.49 \pm 7.1$ & $45.36 \pm 2.98$ & $45.278 \pm 2.8$ & $45.25 \pm 2.89$ & 0.99 \\
K2 (D) & $48.67 \pm 4.17$ & $48.7 \pm 4.1$ & $48.38 \pm 4.05$ & $48.17 \pm 3.74$ & $48.22 \pm 3.83$ & 0.94 \\
K Mean(D) & $46.96 \pm 3.37$ & $46.99 \pm 3.38$ & $46.83 \pm 3.35$ & $46.77 \pm 3.2$ & $46.72 \pm 3.30$ & 0.99 \\
DLD (microns) & 0 & $247.77 \pm 59.21$ & $247.02 \pm 59.08$ & 0 & 0 \\
\hline
\end{tabular}

Abbreviations: Sph, sphere; Cyl, cylinder; SE, spherical equivalent; D, Diopter; UDVA, Uncorrected distance visual acuity; CDVA, Corrected distance visual acuity; ECD, Endothelial cell count; K, Keratometry; DLD, Demarcation line depth.

Table 5 Thinnest Pachymetry Changes of Both Groups at 12 Months

\begin{tabular}{|l|l|l|}
\hline Thinnest Pachymetry (Microns) & Flavin & Peschke-D \\
\hline Preop & $466.78 \pm 34.87$ & $457.5 \pm 35.52$ \\
6 Months & $460.78 \pm 32.61$ & $455.04 \pm 35.16$ \\
I2 Months & $460.1 \pm 32.35$ & $453.76 \pm 36.06$ \\
P value (Pre op-6 months) & 0.006 & 0.06 \\
P value (Pre op-12 months) & 0.002 & 0.01 \\
Percentage change (Pre op-6months) & $1.28 \%$ & $0.53 \%$ \\
Percentage change (Pre op- 12 months) & $1.43 \%$ & $0.81 \%$ \\
\hline
\end{tabular}

group was $247.77 \pm 59.21 \mu$, however, there was no statistically significant difference between the two $(p=0.13)$. Figure 8 shows AS-OCT of one eye each being treated with Flavin and Peschke-D at 1 month and 3 months postoperatively. Beyond 3 months, the demarcation line was patchy and could not be seen prominently in all the eyes.

Flavin

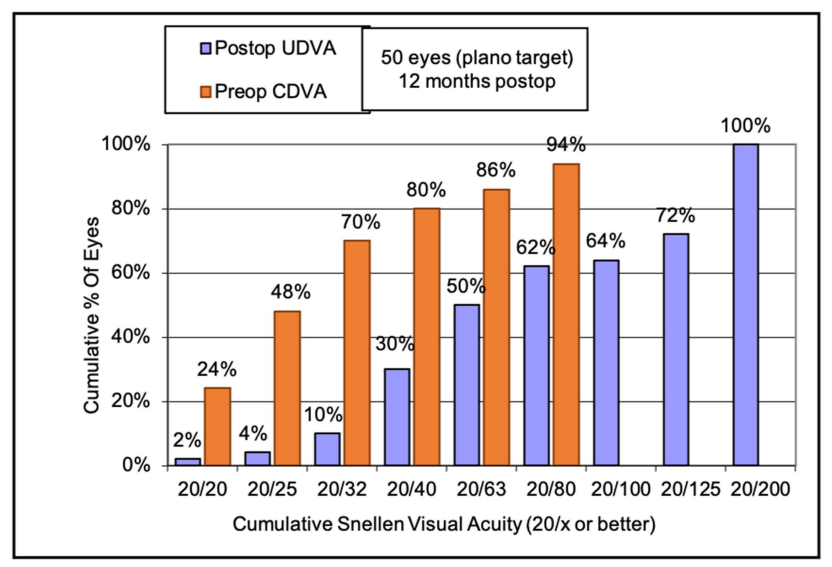

Figure 3 Histogram showing UDVA and CDVA of at 12 months postoperatively.

\section{Postoperative Haze Grading}

Postoperative corneal haze was evaluated using the grading system described by Mohan et al. ${ }^{11}$ At 1 month in the Flavin group, $14 \%$ of eyes had grade 1 and $4 \%$ of eyes had grade 2 haze; whereas, in the Peschke-D group, 18\% of eyes had grade 1 and $2 \%$ of eyes had grade 2 corneal haze. At 12 months, all (100\%) eyes had no or minimal corneal haze grading between $0-0.5$, as shown in Figure 9.

\section{Postoperative Endothelial Cell Density (ECD)}

There was no significant difference in the postoperative ECD values at 12 months compared to preoperative values in either of the groups. The mean postop values of ECD were comparable between the two groups at the last follow-up (Table 2).

Peschke- D

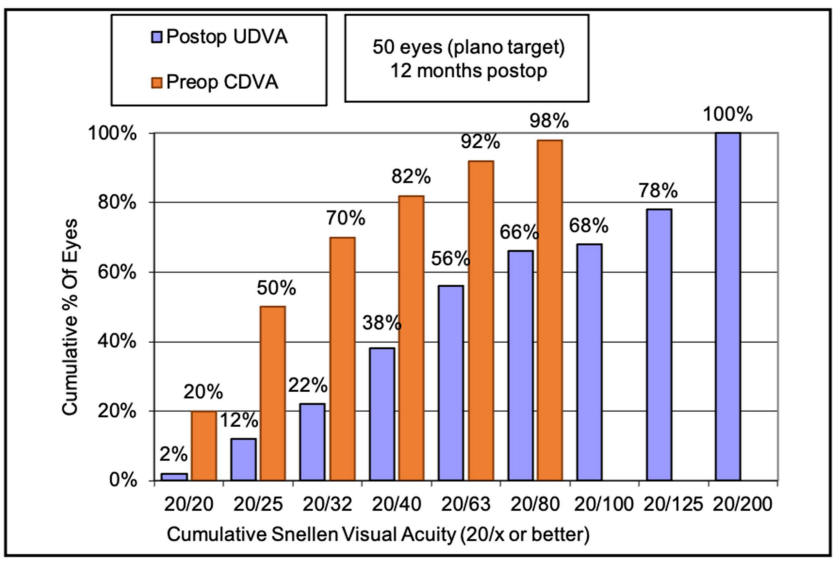


Flavin

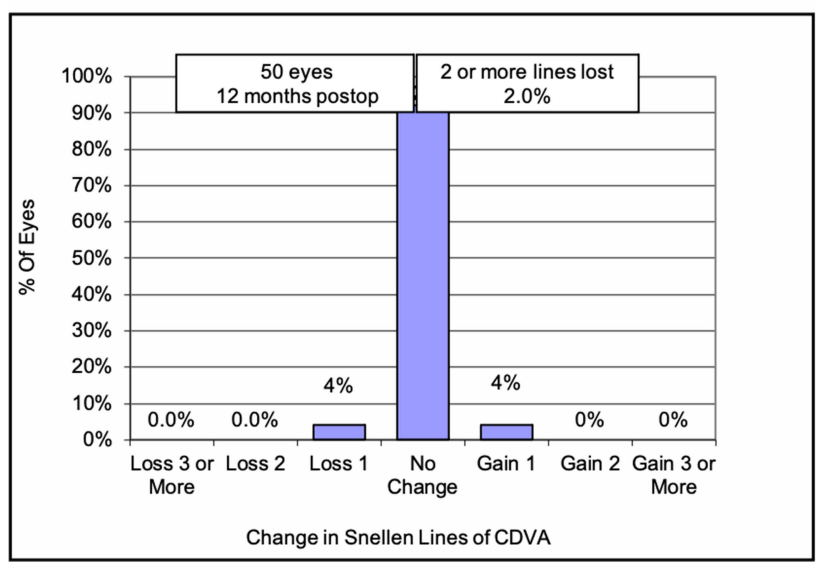

Figure 4 Histogram showing change in Snellen's lines of CDVA.

Flavin

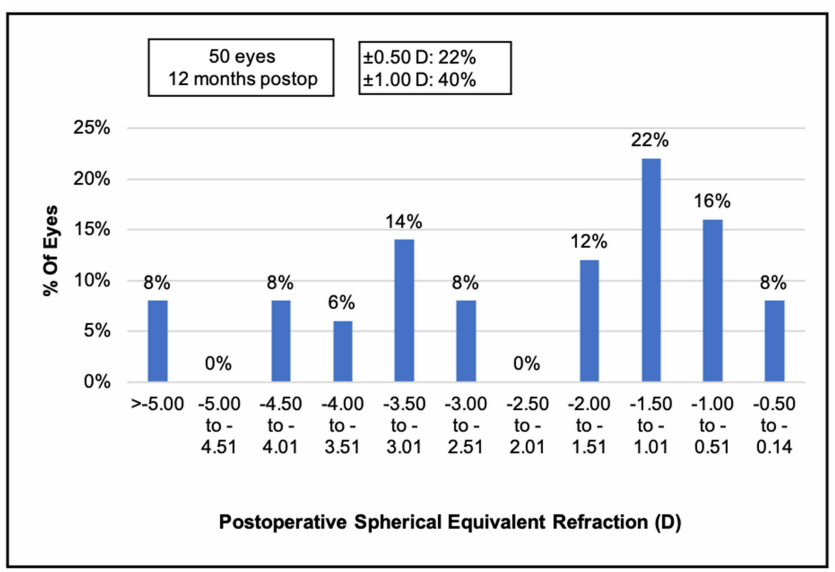

Peschke-D

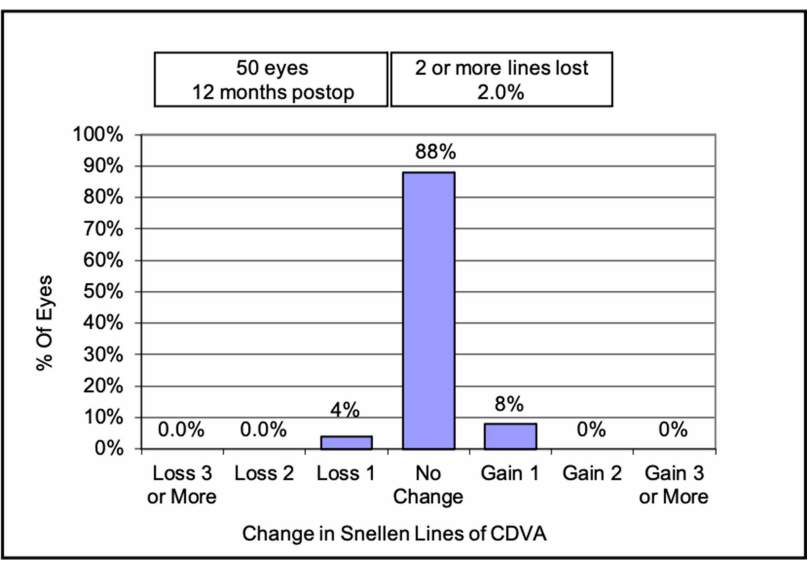

Peschke- D

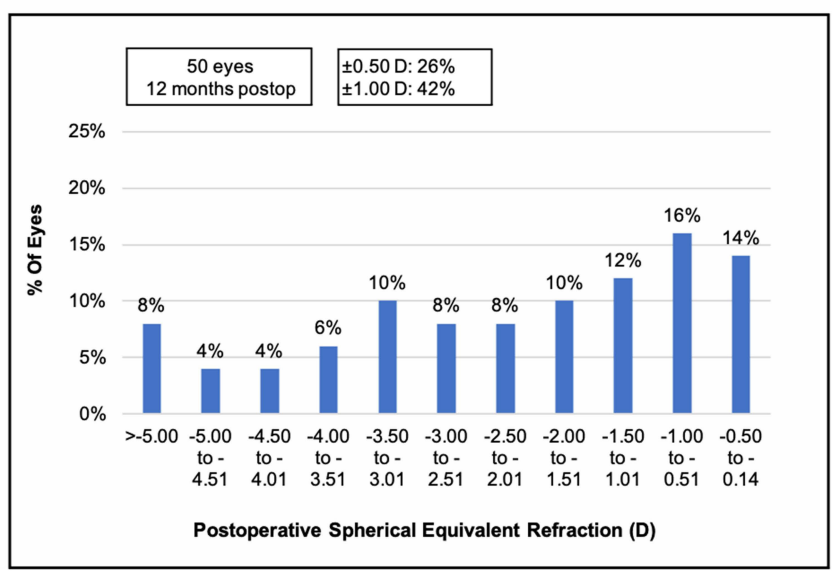

Figure 5 Histogram showing change in spherical equivalent refraction at 12 months postoperatively.

\section{Complications}

No eye in either group had any immediate or long-term postop vision threatening complications such as infectious keratitis, corneal melt, non-resolving corneal oedema or endothelial decompensation.

\section{Discussion}

Collagen cross linking (CXL) is now a proven modality to stabilize progressive keratectasia, the safety and efficacy of which has been established in various published studies. ${ }^{6,7,12}$ Success of the procedure not only involves proper patient selection, right methodology, adequate UVA energy dosage, but also an appropriate composition of photosensitizer agent ie, riboflavin.
Although accelerated cross linking (CXL) has been proposed to be a time saving, safe and effective alternative to conventional Dresden protocol, due to the application of Bunsen-Roscoe law of reciprocity, ${ }^{13-15}$ its efficacy has been questioned in some studies. ${ }^{14,15}$ This is because of the potential depletion of the oxygen, which is required for the formation of the covalent bonds between the collagen fibrils. ${ }^{16,17}$ Hence, in this evaluation study, we decided to perform the conventional Dresden protocol for both the groups, which is a time tested protocol for cross linking.

Results of collagen cross linking using the $0.1 \%$ Peschke riboflavin solution have been published in various studies. Recently, Paningasan et al published their 2 to 3 years after collagen crosslinking results for keratoconus 
Flavin

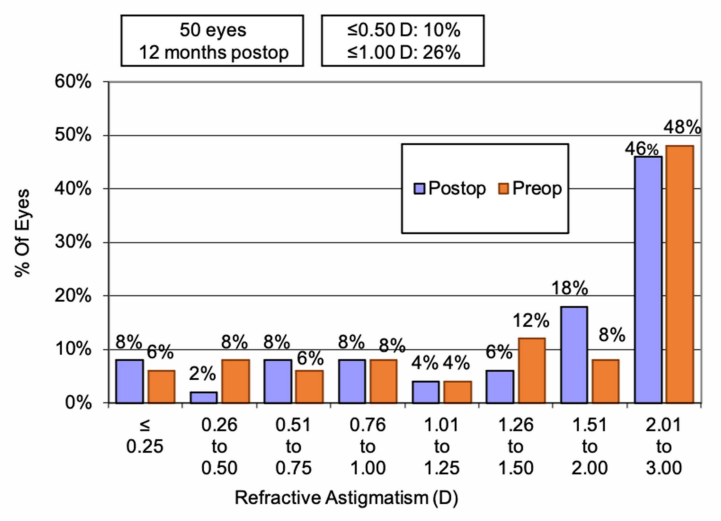

Peschke-D

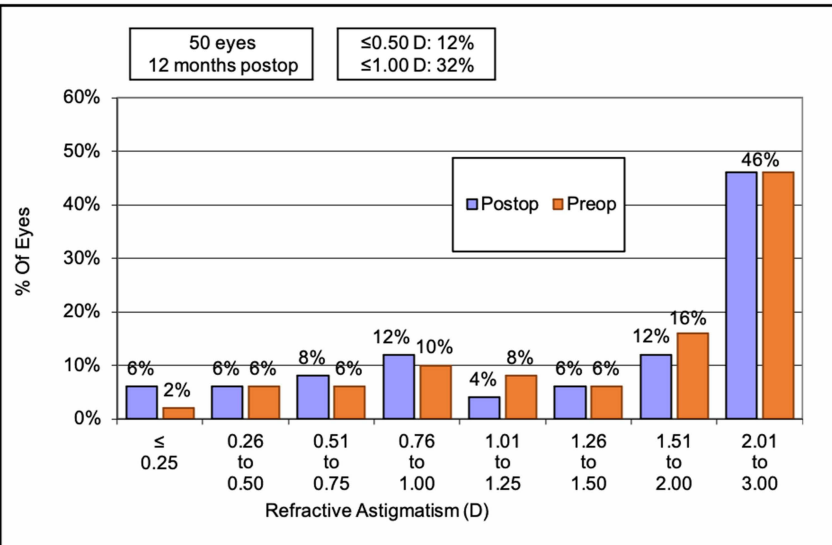

Figure 6 Histogram showing change in refractive astigmatism at 12 months postoperatively.

Flavin

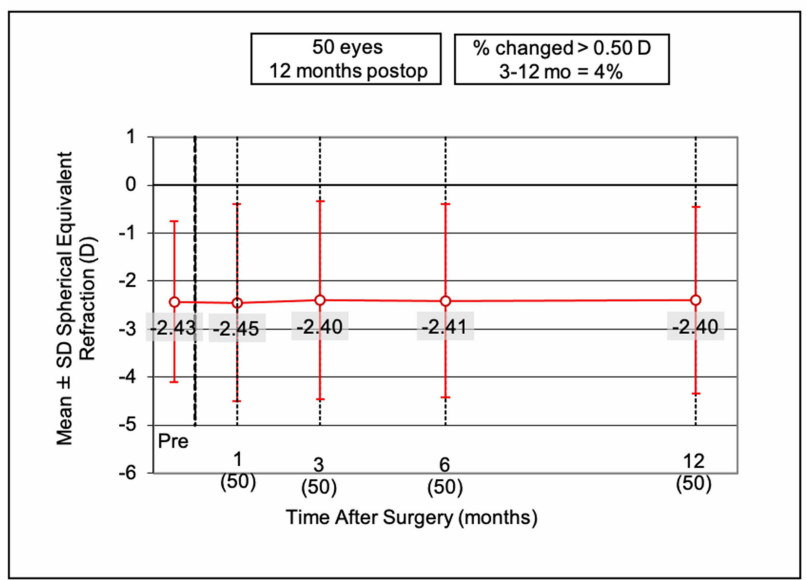

Peschke-D

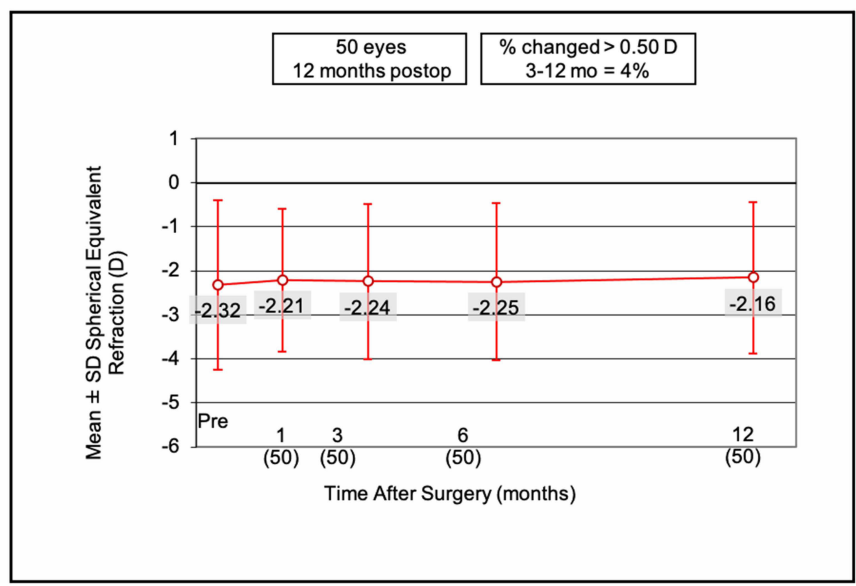

Figure 7 Histogram showing change in mean spherical equivalent at I month, 3 months, 6 months and 12 months postoperatively.

using the Peschke riboflavin solution and standard Dresden protocol, which showed stable outcomes and improvement in corneal curvature parameters and BCVA when compared to the baseline. ${ }^{18}$ In another study by Henriquez et al, who compared 12 month outcomes of standard (Dresden protocol using the $0.1 \%$ riboflavin with 20\% dextran, Peschke) versus trans-epithelial (accelerated protocol using transepithelial riboflavin (Peschke) composed of $0.25 \%$ riboflavin, $1.0 \%$ phosphate hydroxypropyl methylcellulose, and $0.007 \%$ benzalkonium chloride), the authors found both protocols using the two different compositions of dyes comparable in terms of safety, efficacy and keratometric stabilization in pediatric patients with progressive keratoconus at 12 months postop.
No intraoperative or serious postoperative complications occurred in this series of patients. However, one eye had stromal haze that lasted 2 months and resolved with topical corticosteroid treatment. One eye had sterile infiltrates that resolved at postoperative day 22 using standard postoperative treatment regimens. Both eyes were from the epi-off group. In addition, there were progressive cases in both groups ( 2 cases in the A-epi-on group and 3 cases in the epi-off group).

There are several reports in the literature reporting progression in children despite CXL, ${ }^{19-22}$ irrespective of the protocol employed. In adults, however, higher preoperative keratometry values are considered as one of the risk factors for CXL failure. ${ }^{23-25}$ In fact, preoperative keratometry values 
Flavin

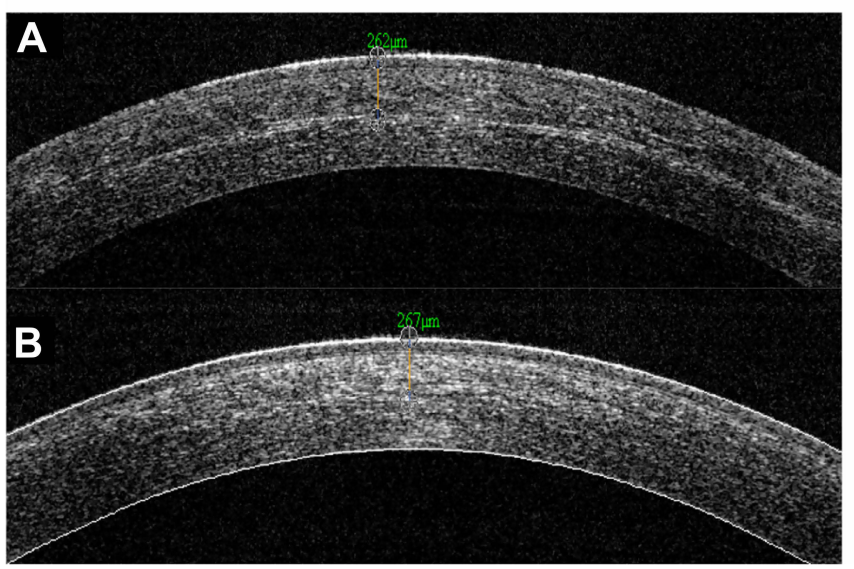

Peschke-D

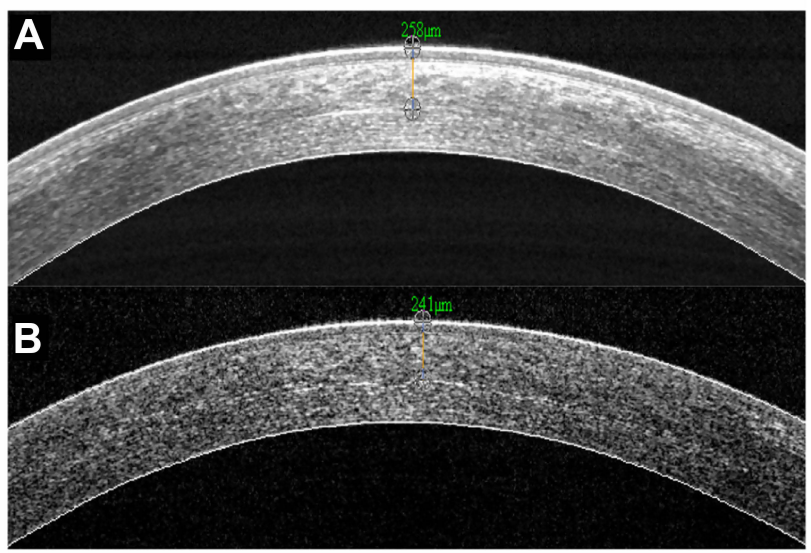

Figure 8 ASOCT image showing demarcation line depth at (A) I-month and (B) 3-months postop for one eye each with Flavin and Peschke-D riboflavin dye.

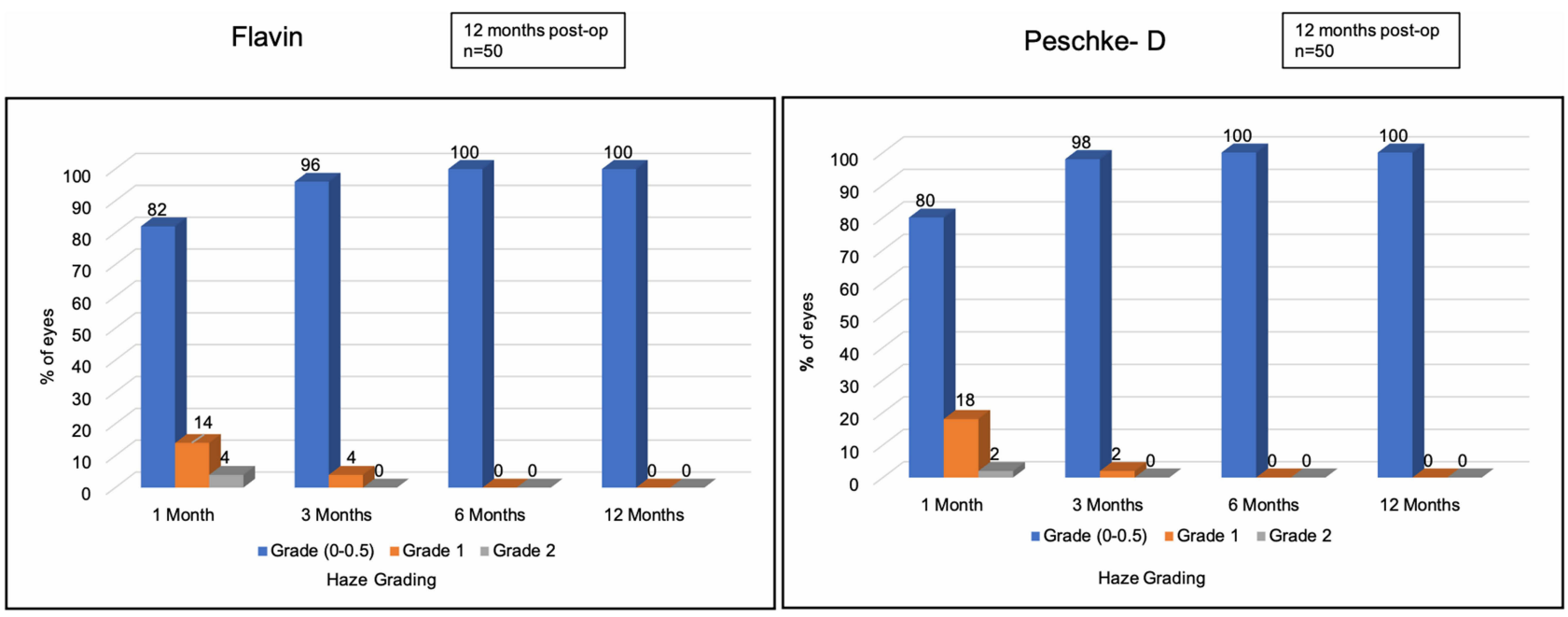

Figure 9 Histogram showing postoperative corneal haze grading.

more than $54 \mathrm{D}$ were shown to be associated with a failure rate up to $12 \%$ for CXL in adults. ${ }^{26}$ In the study by Henriquez et al, 3 of 5 cases that had keratoconus progression had preoperative maximum keratometry more than $54 \mathrm{D}$. In our study, 5 eyes in the Flavin group and 6 eyes in the Peschke group had preoperative maximum keratometry more than 54 D. Despite this, corneal CXL was effective and none of these eyes showed progression beyond $0.5 \mathrm{D}$ in maximum keratometry at 12 months postop. These results are in favor of the effectiveness of CXL for advanced cases of keratoconus, as reported by a previously published study by Sloot et al. ${ }^{24}$ This also indicates similar and equal efficacy of both the comparator riboflavin dyes evaluated in the study in halting the progression in relatively advanced cases of keratoconus.

A pachymetric change of $<2 \%$ within the past 12 months is defined as one of the criteria for progression of keratoconus. ${ }^{27}$ In our study, we found that the mean thinnest pachymetry reduced by $1.43 \%$ and $0.81 \%$ in the Flavin and Peschke groups respectively, indicating an effective stabilization of the disease in both the study groups. As regards the intraoperative pachymetry changes evaluated using ultrasound, both the formulations resulted in a similar degree of reduction in corneal thickness (27\% in Flavin and $26.5 \%$ in Peschke groups). This appears to be comparable to the results published by Rosenblatt et $\mathrm{al}^{28}$ who showed up to $30 \%$ reduction in ultrasound pachymetry during treatment with $0.1 \%$ Peschke riboflavin containing $20 \%$ dextran, which is a similar composition as the Flavin riboflavin evaluated in the study.

The depth of demarcation line has traditionally been linked with the efficacy of the CXL treatment. In our study, both groups had no significant differences in the 
mean depth of demarcation line at 1 month $(256.27 \pm 61.49$ microns in Flavin group and 247.77 \pm 59.21 microns in the Peschke group), which is consistent with the findings of earlier studies published with conventional cross linking using the Dresden protocol. ${ }^{26,29}$

Along with the efficacy, one of our main aims of performing this study was to evaluate the safety of the Flavin, which is a relatively new formulation of riboflavin recently made available in the market. We observed that the safety of the drug was at par with that of Peschke, as none of the eyes treated with Flavin formulation resulted in any sight threatening or long-term side effects or complications such as dense and persistent corneal haze, sterile or infective corneal infiltrates, keratitis, intraocular inflammation, corneal melt or endothelial decompensation.

To conclude, both riboflavin formulations were found to be equally safe and effective in the management of progressive keratoconus in the present randomized comparison study, and resulted in similar changes in terms of keratometry, pachymetry, demarcation line depth and endothelial cell density changes at 12 months postoperatively. To our knowledge, this is the first evaluation of the Flavin riboflavin formulation for the management of progressive keratoconus in a 1 year long randomized comparative study. Further studies with longer term data are suggested to confirm our preliminary findings and establish the long-term safety, efficacy and effects of this formulation on corneal stabilization.

\section{Disclosure}

Dr Sri Ganesh and Dr Sheetal Brar are consultants to Biotech Vision Care, Ahmedabad, India. The study was supported by a limited grant from the Biotech Vision Care Pvt Ltd. The authors report no other conflicts of interest in this work.

\section{References}

1. Wollensak G. Crosslinking treatment of progressive keratoconus: new hope. Curr Opin Ophthalmol. 2006;(17):356-360.

2. Wollensak G, Spoerl E, Seiler T. Riboflavin $0.1 \% /$ ultraviolet-a-induced collagen crosslinking for the treatment of Keratoconus. $\mathrm{Am}$ $J$ Ophthalmol. 2003;135(5):620-627. doi:10.1016/S0002-9394(02) 02220-1

3. Asri D, Touboul D, Fournié P, et al. Corneal collagen crosslinking in progressive keratoconus: multicenter results from the French National Reference Center for Keratoconus. J Cataract Refract Surg. 2011;37 (12):2137-2143. doi:10.1016/j.jcrs.2011.08.026

4. Tan P, Mehta JS. Collagen crosslinking for keratoconus. J Ophthalmic Vis Res. 2011;6(3):153-154.

5. Li G, Fan ZJ, Peng XJ. Corneal collagen crosslinking for conical ectasia of post-LASIK: one-year results. Int J Ophthalmol. 2012;5 (2):190-195. doi:10.3980/j.issn.2222-3959.2012.02.15
6. Derakhshon A, Shandiz JH, Ahadi M, Daneshvar R, Esmaily H. Short-term outcomes of collagen crosslinking for early keratoconus. J Ophthalmic Vis Res. 2011;6(3):155-159.

7. Agrawal V. Long-term results of cornea collagen crosslinking with Riboflavin $0.1 \%$ for Keratoconus. Indian J Ophthalmol. 2013;61 (8):433-434.

8. Sachdev GS, Sachdev M, Recent advances in corneal collagen cross-linking. Indian J Ophthalmol. 2017;65:787-796. doi:10.4103/ ijo.IJO_648_17

9. Goldich Y, Marcovich AL, Barkana Y, Avni I, Zadok D. Safety of corneal collagen cross-linking with UV-A and riboflavin in progressive keratoconus. Cornea. 2010;29(4):409-411. doi:10.1097/ ICO.0b013e3181bd9f8c

10. Galvis V, Tello A, Ortiz AI, Escaf LC. Patient selection for corneal collagen cross-linking: an updated review. ClinOphthalmol. 2017;7 (11):657-668.

11. Mohan RR, Stapleton WM, Sinha S, Netto MV, Wilson SE. A novel method for generating corneal haze in anterior stroma of the mouse eye with the excimer laser. Exp Eye Res. 2008;86(2):235-240. doi:10.1016/j.exer.2007.10.014

12. Jabbar A. Efficacy of corneal collagen crosslinking with riboflavin and ultraviolet a in progressive keratoconus. DJO. 2013;24(1):23-27. doi:10.7869/djo.2013.4

13. Gatzioufas Z, Richoz O, Brugnoli E, Hafezi F. Safety profile of high-fluence corneal collagen cross-linking for progressive keratoconus: preliminary results from a prospective cohort study. $J$ Refract Surg. 2013;29(12):846-848. doi:10.3928/1081597X-20131023-03

14. Lanchares E, Angeles Del Buey M, Cristóbal JA, Lavilla L, Calvo B. Biomechanical property analysis after corneal collagen cross-linking in relation to ultraviolet A irradiation time, Graefes. Arch ClinExpOphthalmol. 2011;249:1223-1227.

15. Schumacher S, Mrochen M, Wernli J, Bueeler M, Seiler T. Optimization model for UV-riboflavin corneal cross-linking. Invest Ophthalmol Vis Sci. 2012;53(2):762-769. doi:10.1167/iovs.11-8059

16. Peyman A, Nouralishahi A, Hafezi F, Kling S, Peyman M. Stromal demarcation line in pulsed versus continuous light accelerated corneal cross-linking for keratoconus. J Refract Surg. 2016;32:206-208. doi:10.3928/1081597X-20160204-03

17. Kling S, Richoz O, Hammer A, et al. Increased biomechanical efficacy of corneal cross-linking in thin corneas due to higher oxygen availability. $J$ Refract Surg. 2015;31:840-846. doi:10.3928/ 1081597X-20151111-08

18. Paningasan JL. Long-term effect on corneal curvature of corneal collagen cross-linking for keratoconus. Philipp $J$ Ophthalmol. 2019;44(2).

19. Buzzonetti L, Petrocelli G. Transepithelial corneal cross-linking in pediatric patients: early results. $J$ Refract Surg. 2012;28:763-767. doi:10.3928/1081597X-20121011-03

20. Shetty R, Nagaraja H, Jayadev C, et al. Accelerated corneal collagen cross-linking in pediatric patients: two-year follow-up results. Biomed Res Int. 2014;2014:894095.

21. Caporossi A, Mazzotta C, Baiocchi S, et al. Age-related long-term functional results after riboflavin UV a corneal crosslinking. J Ophthalmol. 2011;608041.

22. Kumar Kodavoor SK, Arsiwala AZ, Ramamurthy D. One-year clinicalstudy of efficacy of corneal collagen cross-linking in Indian children withprogressive keratoconus. Cornea. 2014;33:919-922. doi:10.1097/ICO.0000000000000197

23. Wollensak G, Iomdina E. Biomechanical and histological changes after corneal crosslinking with and without epithelial debridement. $J$ Cataract Refract Surg. 2009;35:540-546. doi:10.1016/j. jcrs.2008.11.036

24. Sloot F, Soeters N, van der Valk R, et al. Effective corneal collagen crosslinking in advanced cases of progressive keratoconus. $J$ Cataract Refract Surg. 2013;39:1141-1145. doi:10.1016/j. jcrs.2013.01.045 
25. Caporossi A, Mazzotta C, Paradiso AL, et al. Transepithelial cornealcollagen crosslinking for progressive keratoconus: 24-month clinical results. J Cataract Refract Surg. 2013;39:1157-1163. doi:10.1016/j. jcrs.2013.03.026

26. Henriquez MA, Rodríguez AM, Izquierdo L Jr. Accelerated epi-on versus standard epi-off corneal collagen cross-linking for progressive keratoconus in pediatric patients. Cornea. 2017;36(12):1503-1508. doi:10.1097/ICO.0000000000001366

27. Gore DM, Shortt AJ, Allan BD. New clinical pathways for keratoconus. Eye (Lond). 2013;27(3):329-339. doi:10.1038/ eye. 2012.257
28. Rosenblat E, Hersh PS. Intraoperative corneal thickness change and clinical outcomes after corneal collagen crosslinking: standard crosslinking versus hypotonic riboflavin. J Cataract Refract Surg. 2016;42 (4):596-605. doi:10.1016/j.jcrs.2016.01.040

29. Kymionis GD, Tsoulnaras KI, Liakopoulos DA, et al. Corneal stromal demarcation line depth following standard and a modified high intensity corneal cross-linking protocol. $J$ Refract Surg. 2016;32:218-222. doi:10.3928/1081597X-20160216-01

\section{Publish your work in this journal}

Clinical Ophthalmology is an international, peer-reviewed journal covering all subspecialties within ophthalmology. Key topics include: Optometry; Visual science; Pharmacology and drug therapy in eye diseases; Basic Sciences; Primary and Secondary eye care; Patient Safety and Quality of Care Improvements. This journal is indexed on PubMed

Submit your manuscript here: https://www.dovepress.com/clinical-ophthalmology-journal
Central and CAS, and is the official journal of The Society of Clinical Ophthalmology (SCO). The manuscript management system is completely online and includes a very quick and fair peer-review system, which is all easy to use. Visit http://www.dovepress.com/ testimonials.php to read real quotes from published authors. 